\title{
Gain Scheduling Resonant Control with Anti-Windup Compensation for LPV Systems *
}

\author{
Gabriel P. Flores * Jeferson V. Flores * \\ João M. Gomes da Silva Jr. * Aurélio T. Salton* \\ * Escola de Engenharia, Universidade Federal do Rio Grande do Sul, \\ Porto Alegre, Brazil (e-mail:\{gabriel.pascottini, jeferson.flores, \\ jmgomes, aurelio.salton\}@ufrgs.br)
}

\begin{abstract}
In this paper, we address the problem of periodic reference tracking and disturbance rejection under control saturation for linear parameter-varying - LPV systems robust to small period variations. The proposed controller is based on a modified resonant structure and a gain scheduling state-feedback, while saturation effects are mitigated through a scheduled anti-windup compensator. Control design is carried out by the solution of an optimization problem under matrix inequalities constraints that ensure closed-loop stability and performance. In addition, it is possible to determine admissible sets for both initial conditions and references/disturbances amplitudes for which tracking/rejection is guaranteed with a bounded steady-state tracking error. A numerical example illustrates the method.
\end{abstract}

Keywords: Robust control, Systems with saturation, Linear parameter varying system, anti-windup

\section{INTRODUCTION}

Tracking and rejection of periodic signals is a research field of crescent interest to many engineering applications, e.g. mechanical load reduction in offshore platforms (Zhang et al., 2017) and active vehicle suspensions (Tseng and Hrovat, 2015). For problems where the signals of interest have precisely known and time-invariant frequencies, the internal model principle - IMP based controllers, such as resonant or repetitive control (Pereira et al., 2014; Hara et al., 1988), provide theoretical guarantees of perfect reference/disturbance tracking/rejection in steady-state. However, for applications where these frequencies are uncertain or time-varying, the standard IMP approaches exhibit degraded tracking performance (Steinbuch, 2002). A well established solution to this problem is the use of highorder repetitive controllers, where several delay elements are combined to enlarge the high-gain region around the nominal frequency (Steinbuch, 2002). However, the use of additional delay elements may lead to stability and robustness problems ( $\mathrm{Li}$ and $\mathrm{Ye}, 2018$ ). Other alternative is the modified resonant structure proposed in Flores et al. (2016), where a notch filter is employed in series with a resonant controller to enlarge the high-gain region around the nominal resonance peaks.

Furthermore, there are several applications where the plant is not only subject to frequency variations in the references/disturbances, but it also varies with time. Depending on the system structure, these plants can be modeled

\footnotetext{
* This study was financed in part by the Coordenação de Aperfeiçoamento de Pessoal de Nível Superior - Brasil (CAPES) - Finance Code 001 and CNPq (Brazil), grant numbers: PQ-306223/2018-0, PQ-309272/2015-7, PQ-305979/2015-9, Univ-422992/2016-0.
}

as linear parameter varying (LPV) systems (Hoffmann and Werner, 2015). This particular structure can be employed to model nonlinear plants in the so called quasi-LPV approach (Rugh and Shamma, 2000), by converting the system's nonlinearities to a linear parameter-dependent structure valid in a certain region of the state-space. Example of plants that can be modeled as LPV/quasi-LPV problems may range from mechanical load reduction in wind turbines (Castro et al., 2017) to state estimation in vehicles (Kang et al., 2018).

On the other hand, almost every actuator has intrinsic limitations which leads to the problem of control saturation (Tarbouriech et al., 2011). In this context, restrictions on the allowable control signal can significantly deteriorate transient performance, in the phenomenon of windup (Tarbouriech and Turner, 2009), or even lead the closed-loop system to instability. In terms of steady-state tracking performance, saturation imposes constraints on the amplitude of references/disturbances signals and initial conditions for which the tracking/rejection is guaranteed (Flores et al., 2009). Regarding IMP based controllers able to deal with tracking/rejection of periodic signals taking into account small frequency variation and input saturation we can cite (Ramos and Costa-Castelló, 2013) and (Flores and Flores, 2018).

In this work, we propose a solution for the tracking/rejection problem of LPV multiple-input multipleoutput (MIMO) systems subject to input saturation and periodic references/disturbances with time-varying or uncertain frequencies. A scheduled static anti-windup compensator is added to the modified resonant state-space formulation presented in Flores et al. (2016) and a gain- 
scheduling feedback of the plant and controller states is considered to stabilize the closed-loop system. Conditions in a quasi-linear matrix inequality (LMI) form ${ }^{1}$ are derived to ensure closed-loop stability and performance. An optimization problem aiming to maximize the set of admissible initial states or the allowable reference/disturbance amplitudes is also provided. A numerical example illustrate the proposed method.

Notation: The transpose of $A$ is represented by $A^{\prime}$. The $i$-th component of a vector $x$ is denoted by $x_{(i)}$, $A_{(i)}$ represents the $i$-th row of a matrix $A \in \mathbb{R}^{n \times m}$. $\operatorname{diag}\left\{A_{1}, A_{2}\right\}$ is a block diagonal matrix obtained from $A_{1}$ and $A_{2}, 0_{n \times m}$ is the $n \times m$ null matrix. * represents symmetric block elements in a matrix, while $H e\{A W\}=$ $A W+W^{\prime} A^{\prime} . \operatorname{Co}\{\}$ denotes a convex hull. $I$ is the identity matrix with appropriate dimensions.

\section{PRELIMINARIES}

\subsection{Open Loop-System}

Consider the following LPV continuous-time system

$$
\begin{aligned}
\dot{x}(t) & =A(\sigma(t)) x(t)+B_{s a t} u_{u_{0}}(u(t))+B_{d} d(t), \\
y(t) & =C(\sigma(t)) x(t), \\
e(t) & =r(t)-y(t),
\end{aligned}
$$

where $x(t) \in \mathbb{R}^{n}$ is the state vector, $u(t) \in \mathbb{R}^{m}$ is the plant input, $y(t) \in \mathbb{R}^{p}$ is the plant output, $d(t) \in \mathbb{R}^{l}$ represents a disturbance, $r(t) \in \mathbb{R}^{p}$ is a reference vector and $e(t) \in \mathbb{R}^{p}$ is the tracking error.

The saturation nonlinearity presented in (1) is described by $\operatorname{sat}_{u_{0_{(i)}}}\left(u_{(i)}(t)\right) \triangleq \operatorname{sgn}\left(u_{(i)}(t)\right) \min \left(\left|u_{(i)}(t)\right|, u_{0_{(i)}}\right)$, for $i=1, \cdots, m$, where $u_{0_{(i)}}$ is the $i$-th input saturation limit and $u_{(i)}(t)$ is the $i$-th plant input.

The vector of $N$ time-varying parameters is represented by $\sigma(t)=\left[\begin{array}{llll}\sigma_{1}(t) & \sigma_{2}(t) & \cdots & \sigma_{N}(t)\end{array}\right]^{\prime}$, where every component of $\sigma(t)$ is assumed to be measurable and limited in amplitude such that

$$
\underline{\sigma}_{j} \leq \sigma_{j}(t) \leq \bar{\sigma}_{j}, j=1, \cdots, N
$$

It is also considered that $\left|\dot{\sigma}_{j}(t)\right|<\infty \forall t$. Then, we can adopt a polytopic description for $\sigma(t)$ such that

$$
\sigma(t) \in \mathcal{B}=\operatorname{Co}\left\{v_{1}, v_{2}, \cdots, v_{2^{N}}\right\}
$$

i.e.,

$$
\sigma(t)=\sum_{g=1}^{2^{N}} \mu_{g}(t) v_{g}, \sum_{g=1}^{2^{N}} \mu_{g}(t)=1,0 \leq \mu_{g}(t) \leq 1
$$

where $v_{g}, g=1, \cdots, 2^{N}$ are vertices derived from (2).

Each element of vectors $r(t)$ and $d(t)$ are assumed to be periodic with uncertain/time-varying fundamental frequencies given by $\omega_{r}(t)=\left[1+\delta_{r}(t)\right] \omega_{0 r},\left|\delta_{r}(t)\right| \leq \bar{\delta}_{r}$, $\omega_{d}(t)=\left[1+\delta_{d}(t)\right] \omega_{0 d}, \quad\left|\delta_{d}(t)\right| \leq \bar{\delta}_{d}$ where $\bar{\delta}_{r}$ and $\bar{\delta}_{d}$ denote the maximum percent deviation around $\omega_{0 r}$ and $\omega_{0 d}$, respectively.

\subsection{Controller Description}

Consider the modified resonant controller approach presented in Flores et al. (2016), referred as notch-resonant

\footnotetext{
1 LMIs provided that some scalar parameters are fixed.
}

controller - NRC. In this reference, the control structure is composed of the cascade connection between a notch filter and a resonant structure, enlarging the high-gain region around the nominal frequency and maintaining the $-40 \mathrm{~dB} / \mathrm{dec}$ roll-off from the original resonant controller. Hence, in order to compensate $M$ desired frequencies, the NRC structure is given by:

$$
G_{m n r}(s)=\prod_{k=1}^{M}\left(\frac{s^{2}+2 \zeta_{z} \omega_{k} s+\omega_{k}^{2}}{s^{2}+2 \zeta_{h} \omega_{k} s+\omega_{k}^{2}} \cdot \frac{\omega_{k}^{2}}{s^{2}+\omega_{k}^{2}}\right)
$$

with $\zeta_{h}<\zeta_{z}<1$. A study regarding the effects of different values for $\zeta_{h}$ and $\zeta_{z}$ is presented in Flores et al. (2016), where the maximum "enlargement" of the high-gain region is obtained for $\zeta_{h}=0.01$ and $\zeta_{z}=0.99$.

Controller (5) can be represented in state-space form as an augmented state representation of the resonant controller and the notch filter. The notch filter representation is given by

$$
\begin{aligned}
& \dot{x}_{n k}(t)=A_{n k} x_{n k}(t)+B_{n} e_{\varepsilon}(t) \\
& y_{n k}(t)=C_{n k} x_{n k}(t)+D_{n} e_{\varepsilon}(t)
\end{aligned}
$$

where $x_{n k}(t) \in \mathbb{R}^{2}$ is the notch filter's state vector, $e_{\varepsilon}(t)=$ $r_{\varepsilon}(t)-y_{\varepsilon}(t), \varepsilon=1, \cdots, p$ is the $\varepsilon$-th error channel and

$$
\begin{aligned}
& A_{n k}=\left[\begin{array}{cc}
0 & \omega_{k} \\
-\omega_{k} & -2 \zeta_{h} \omega_{k}
\end{array}\right], B_{n}=\left[\begin{array}{l}
0 \\
1
\end{array}\right] \\
& C_{n k}=\left[\begin{array}{ll}
0 & 2 \omega_{k}\left(\zeta_{z}-\zeta_{h}\right)
\end{array}\right], D_{n}=1 .
\end{aligned}
$$

The resonant controller in series with the filter is represented by

$$
\begin{aligned}
& \dot{x}_{r k}(t)=A_{r k} x_{r k}(t)+B_{r} y_{n k}(t) \\
& y_{r k}(t)=C_{r} x_{r k}(t)
\end{aligned}
$$

with

$$
A_{r k}=\left[\begin{array}{cc}
0 & \omega_{k} \\
-\omega_{k} & 0
\end{array}\right], B_{r}=\left[\begin{array}{l}
0 \\
1
\end{array}\right], C_{r}=\left[\begin{array}{c}
\omega_{k} \\
0
\end{array}\right]^{\prime} .
$$

Based on (6) and (7), the augmented state vector $x_{k}(t)=$ $\left[x_{r k}(t)^{\prime} x_{n k}(t)^{\prime}\right]^{\prime} \in \mathbb{R}^{4}$ results in

$$
\begin{aligned}
\dot{x}_{k}(t) & =A_{k} x_{k}(t)+\bar{B} e_{\varepsilon}(t) \\
y_{k}(t) & =x_{k}(t)
\end{aligned}
$$

with

$$
A_{k}=\left[\begin{array}{cc}
A_{r k} & B_{r} C_{n k} \\
0_{2 \times 2} & A_{n k}
\end{array}\right], \bar{B}=\left[\begin{array}{c}
B_{r} D_{n} \\
B_{n}
\end{array}\right] .
$$

Finally, a parallel interconnection of $M$ NRCs, each tunned for a frequency $\omega_{k}, k=1, \cdots, M$ is given by

$$
\dot{x}_{m n}(t)=A_{m n} x_{m n}(t)+B_{m n} e_{\varepsilon}(t)
$$

with $A_{m n}=\operatorname{diag}\left\{A_{1}, \cdots, A_{M}\right\}$ and $B_{m n}=\left[\bar{B}^{\prime} \cdots \bar{B}^{\prime}\right]^{\prime}$.

Note that the MIMO nature of (1) must be taken into account, hence, to verify the IMP, the controller (9) must be inserted on each output error channel, which leads to

$$
\dot{x}_{c}(t)=A_{c} x_{c}(t)+B_{c} e(t)
$$

where $x_{c}(t) \in \mathbb{R}^{n_{c}}, n_{c}=4 M p$,

$$
A_{c}=\operatorname{diag}\{\overbrace{A_{m n}, \cdots, A_{m n}}^{p-\text { tuple }}\}, B_{c}=\operatorname{diag}\{\overbrace{B_{m n}, \cdots, B_{m n}}^{p-\text { tuple }}\} \text {. }
$$

At a given frequency $\omega_{k}$, it is also assumed that there is no zero-pole cancellation between the controller and plant $\forall \sigma(t) \in \mathcal{B}, t \geq 0$, otherwise the mode corresponding to $\omega_{k}$ should be inserted multiple times until no cancellation occurs. 


\subsection{Closed-Loop Formulation}

To mitigate the loss of transient performance caused by the control saturation and also to add another degree of freedom to the design problem, an scheduled static antiwindup compensator is added to the controller dynamics (10) as follows

$$
\dot{x}_{c}(t)=A_{c} x_{c}(t)+B_{c} e(t)-B_{c} E_{c}(\sigma(t)) \psi_{u_{0}}(u(t)),
$$

where $E_{c}(\sigma(t))$ is the anti-windup gain to be determined and $\psi_{u_{0}}(u(t))$ is a dead-zone nonlinearity given by

$$
\psi_{u_{0}}(u(t))=u(t)-s a t_{u_{0}}(u(t)) .
$$

To stabilize the closed-loop system, consider a scheduling state feedback control law defined as

$$
u(t)=F_{s}(\sigma(t)) x(t)+F_{c}(\sigma(t)) x_{c}(t)=F(\sigma(t)) z(t),
$$

with $z(t)$ being the augmented state vector $z(t)=$ $\left[x(t)^{\prime} x_{c}(t)^{\prime}\right]^{\prime} \in \mathbb{R}^{(n+n c)}$ and $F(\sigma(t))=\left[F_{s}(\sigma(t)) F_{c}(\sigma(t))\right]$. The complete closed-loop structure is shown in Fig. 1.

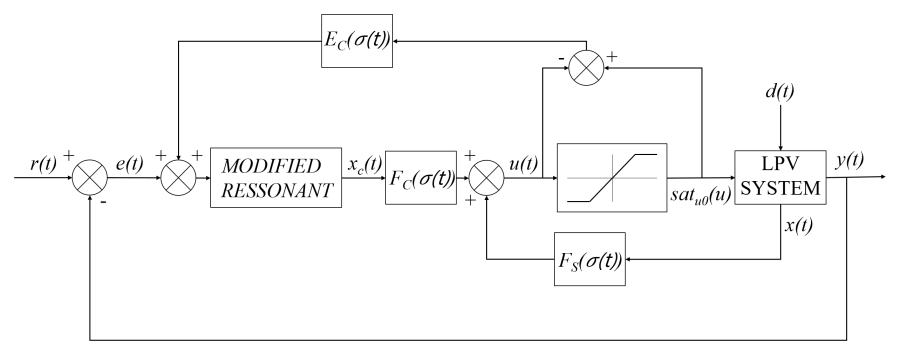

Figure 1. Closed-loop system scheme.

Combining (1), (11) and (12) results in the following closed-loop dynamics

$$
\begin{aligned}
\dot{z}(t)= & \left(A_{a}(\sigma(t))+B_{a} F(\sigma(t)) z(t)\right. \\
& -\left(B_{a}+V E_{c}(\sigma(t))\right) \psi_{u_{0}}(u(t))+B_{q} q(t),
\end{aligned}
$$

where $q(t)=\left[r(t)^{\prime} d(t)^{\prime}\right]^{\prime}$ is the vector of exogenous signals and

$$
\begin{aligned}
A_{a}(\sigma(t)) & =\left[\begin{array}{cc}
A(\sigma(t)) & 0_{n \times n_{c}} \\
-B_{c} C(\sigma(t)) & A_{c}
\end{array}\right], B_{a}=\left[\begin{array}{c}
B \\
0_{n_{c} \times m}
\end{array}\right], \\
B_{q} & =\left[\begin{array}{cc}
0_{n \times p} & B_{d} \\
B_{c} & 0_{n_{c} \times l}
\end{array}\right], V=\left[\begin{array}{c}
0_{n \times p} \\
B_{c}
\end{array}\right] .
\end{aligned}
$$

Considering now a linear dependence of $A_{a}(\sigma(t)), F(\sigma(t))$ and $E_{c}(\sigma(t))$ with $\sigma(t)$, then, from (4), if follows that

$$
\begin{aligned}
& A_{a}(\sigma(t))=\sum_{g=1}^{2^{N}} \mu_{g}(t) A_{a_{g}}, F(\sigma(t))=\sum_{g=1}^{2^{N}} \mu_{g}(t) F_{g}, \\
& E_{c}(\sigma(t))=\sum_{g=1}^{2^{N}} \mu_{g}(t) E_{c_{g}}
\end{aligned}
$$

with $A_{a_{g}}, F_{g}$ and $E_{c_{g}}$ being matrices $A_{a}(\sigma(t)), F(\sigma(t))$ and $E_{c}(\sigma(t))$ evaluated at each vertex $v_{g}$ of $\mathcal{B}$, i.e. $A_{a_{g}}=$ $A_{a}\left(v_{g}\right), F_{g}=F\left(v_{g}\right)$ and $E_{c_{g}}=E_{c}\left(v_{g}\right)$.

\section{CONTROLLER AND ANTI-WINDUP DESIGN}

\subsection{Problem Statement}

Tracking and rejection of periodic signals for input saturating linear systems is challenging since the closed-loop system (13) exhibits equilibrium trajectories instead of equilibrium points. In addition, the maximum values of reference/disturbances must be admissible, i.e., the saturation limits should not be violated in steady-state. To address these issues, it is proposed an ultimate boundedness framework based on the following sets (Flores et al., 2009): a set of initial conditions $\mathcal{Z}_{0}$; a set of admissible references/disturbances signals $\mathcal{Q}_{0}$ and a positively invariant terminal set $\mathcal{J}_{0} \subset \mathcal{Z}_{0}$ included in the closed-loop linearity region $S\left(F, u_{0}\right) \triangleq\left\{z \in \mathbb{R}^{n+n_{c}} ;|F z| \leq u_{0}\right\}$. Hence, since inside $\mathcal{J}_{0}$ the closed-loop system is linear, then the tracking/rejection is ultimately guaranteed by the IMP.

Regarding the LPV problem, the plant varying structure includes another level of complexity because stability and performance must be ensured $\forall \sigma(t) \in \mathcal{B}$. In this case, a possible solution is to include the set $\mathcal{J}_{0}$ in the intersection of the linearity regions given by gains $F_{g}, g=1, \cdots 2^{N}$, i.e., $\mathcal{J}_{0} \in \bigcap_{g=1}^{2^{N}} S\left(F_{g}, u_{0}\right)$. Then, it follows that in this set the closed-loop system is linear (no saturation occurs) for all $F(\sigma(t))$, such that $\sigma(t) \in \mathcal{B}$.

In this framework, we stated the following problem:

Problem 1. Compute scheduled gains $F(\sigma(t))$ and $E_{c}(\sigma(t))$ such that, $\forall \sigma(t) \in \mathcal{B}, \forall z(0) \in \mathcal{Z}_{0}$ and $\forall q(t) \in \mathcal{Q}_{0}$, the corresponding trajectories of (13) are uniformly ultimately bounded to the set $\mathcal{J}_{0} \in \bigcap_{g=1}^{2^{N}} S\left(F_{g}, u_{0}\right)$.

As in (Flores et al., 2009), these sets are assumed to be ellipsoids defined as:

$$
\begin{aligned}
& \mathcal{Z}_{0}=\Omega(P, 1)=\left\{z \in \mathbb{R}^{n+n_{c}} ; z^{\prime} P z \leq 1\right\}, P=P^{\prime}>0, \\
& \mathcal{J}_{0}=\Omega(\eta P, 1)=\left\{z \in \mathbb{R}^{n+n_{c}} ; z^{\prime} \eta P z \leq 1\right\}, \eta>1, \\
& \mathcal{Q}_{0}=\Omega(R, 1)=\left\{q \in \mathbb{R}^{p+l} ; q^{\prime} R q \leq 1\right\}, R=R^{\prime}>0 .
\end{aligned}
$$

Note that $\mathcal{J}_{0}$ is a scaled version of $\mathcal{Z}_{0}$ with $\eta$ being the scaling factor to be determined.

Regarding Problem 1, the following optimization subproblems can thus be considered: i) maximization of $\mathcal{Z}_{0}$ for a given $\mathcal{Q}_{0}$; ii) maximization of $\mathcal{Q}_{0}$ for a given $\mathcal{Z}_{0}$; iii) joint maximization of $\mathcal{Z}_{0}$ and $\mathcal{Q}_{0}$.

\subsection{Stability Conditions}

The following lemma establish a modified sector condition for the dead-zone nonlinearity and the scheduled gain $F(\sigma(t))$ :

Lemma 2. Consider a matrix $G(\sigma(t)) \in \mathbb{R}^{m \times n+n c}$. If $z(t)$ is such that

$$
S\left(F, G, u_{0}\right)=\left\{z \in \mathbb{R}^{n+n_{c}} ;\left|(F-G)_{(i)} z\right| \leq u_{0_{(i)}}\right\}
$$

for $i=1, \cdots, m$, then

$$
\psi_{u_{0}}(F(\sigma(t)) z(t))^{\prime} T\left(\psi_{u_{0}}(u(t))+G(\sigma(t)) z(t)\right) \leq 0 .
$$

is satisfied for any diagonal positive definite matrix $T \in$ $\mathbb{R}^{m \times m}$

The solution of Problem 1 can therefore be addressed by the following theorem:

Theorem 3. If there are symmetric positive-definite matrices $W \in \mathbb{R}^{\left(n+n_{c}\right) \times\left(n+n_{c}\right)}$ and $R \in \mathbb{R}^{(p+l) \times(p+l)}$, matrices 
$Y_{g} \in \mathbb{R}^{m \times\left(n+n_{c}\right)}, X_{g} \in \mathbb{R}^{m \times\left(n+n_{c}\right)}, M_{g} \in \mathbb{R}^{p \times m}$, diagonal matrices $L_{g} \in \mathbb{R}^{m \times m}$ and scalars $\lambda>0$ and $\eta>1$ that satisfy

$$
\begin{gathered}
{\left[\begin{array}{ccc}
\Gamma & -B_{a} L-V M_{g}+X_{g}^{\prime} & B_{q} \\
* & -2 L & 0 \\
* & * & -\lambda R
\end{array}\right]<0} \\
{\left[\begin{array}{cc}
W & * \\
Y_{g_{(i)}}-X_{g_{(i)}} & u_{0_{(i)}}^{2}
\end{array}\right] \geq 0, i=1, \cdots, m} \\
{\left[\begin{array}{lc}
\eta W & * \\
Y_{g_{(i)}} & u_{0_{(i)}}^{2}
\end{array}\right] \geq 0, i=1, \cdots, m}
\end{gathered}
$$

for all $g=1,2, \cdots, 2^{N}$, with $\Gamma=H e\left\{A_{a_{g}} W+B_{a} Y_{g}\right\}+$ $\bar{\lambda} W, \bar{\lambda}=\lambda(-1+3 \eta)$, then the control law (12) with gains $F_{g}=Y_{g} W^{-1}$ and $E_{c_{g}}=M_{g} L^{-1}$ ensure $\forall q(t) \in \Omega(R, 1)$ that all trajectories of $(13)$ starting in $\Omega(P, 1)$ converge to $\Omega(\eta P, 1)$ where $P=W^{-1}$ and that $\Omega(\eta P, 1)$ is a positively invariant set inside the intersection of linearity regions $S\left(F_{g}, u_{0}\right)$.

Proof. The proof follows the same ideas as in (Flores et al., 2009 ) employing the modified sector condition presented in Lemma 2. Consider $\mathbb{A}(t)=A_{a}(\sigma(t))+B_{a} F(\sigma(t)), \mathbb{B}(t)=$ $B_{a}+V E_{c}(\sigma(t))$ and a quadratic Lyapunov candidate function $\mathbf{V}(z(t))=z(t)^{\prime} P z(t)$. Therefore, the derivative of $\mathbf{V}(z(t))$ along the trajectories of (13) results in

$$
\begin{aligned}
\dot{\mathbf{V}}(z(t))= & z(t)^{\prime}\left(\mathbb{A}(t)^{\prime} P+P \mathbb{A}(t)\right) z(t)+2 z(t)^{\prime} P B_{q} q(t) \\
& -2 z(t)^{\prime} P \mathbb{B}(t) \psi_{u_{0}}(F(\sigma(t)) z(t)) .
\end{aligned}
$$

From Lemma 2, if $z(t) \in S\left(F(\sigma(t)), G(\sigma(t)), u_{0}\right) \forall t$, then (16) is verified. In this case,

$$
\begin{aligned}
\dot{\mathbf{V}}(z(t)) & -2 \psi_{u_{0}}(F(\sigma(t)) z(t))^{\prime} T \psi_{u_{0}}(F(\sigma(t)) z(t)) \\
& +2 \psi_{u_{0}}(F(\sigma(t)) z(t))^{\prime} T G(\sigma(t)) z(t)<0
\end{aligned}
$$

readily implies $\dot{\mathbf{V}}(z(t))<0$.

The ultimately bounded approach described in section 3.1 can be implemented assuming that $(20)$ is verified $\forall z(t) \in$ $\Omega(P, 1) \subset S\left(F(\sigma(t)), G(\sigma(t)), u_{0}\right), \forall z(t) \notin \Omega(\eta P, 1)$ and for all admissible $q(t) \in \Omega(R, 1)$. This implies that all trajectories of (13) starting in $\Omega(P, 1)$ converge to $\Omega(\eta P, 1)$ in a finite time $t_{1}$ and remain inside this inner set $\forall t \geq t_{1}$. Using the S-procedure as proposed in (Flores et al., 2009), this is possible if exists a positive scalar $\lambda$, such that

$$
\begin{aligned}
\dot{\mathbf{V}}(z(t)) & -2 \psi_{u_{0}}(F(\sigma(t)) z(t))^{\prime} T \psi_{u_{0}}(F(\sigma(t)) z(t)) \\
& +2 \psi_{u_{0}}(F(\sigma(t)) z(t))^{\prime} T G(\sigma(t)) z(t) \\
& +\lambda\left(1-z(t)^{\prime} P z(t)\right)+3 \lambda\left(z(t)^{\prime} \eta P z(t)-1\right) \\
& +\lambda\left(1-q(t)^{\prime} R q(t)<0 .\right.
\end{aligned}
$$

Hence, writing the right hand side of (21) as

$$
\begin{aligned}
& {\left[z(t)^{\prime} \quad \psi_{u_{0}}(F(\sigma(t)) z(t))^{\prime}\right.} \\
& \left.q(t)^{\prime}\right] \mathcal{M}\left[\begin{array}{c}
z(t) \\
\left.\psi_{u_{0}}(F(\sigma)) z(t)\right) \\
q(t)
\end{array}\right], \\
& \mathcal{M}=\left[\begin{array}{ccc}
\bar{\Gamma}-P \mathbb{B}(t)+G(\sigma(t))^{\prime} T & P B_{q} \\
* & -2 T & 0 \\
* & * & -\lambda R
\end{array}\right]
\end{aligned}
$$

with $\bar{\Gamma}=\mathbb{A}(t)^{\prime} P+P \mathbb{A}(t)+\bar{\lambda} P$, it suffices that $\mathcal{M}<0$ to guarantee that $(21)$ is satisfied. Pre- and post-multiplying $\mathcal{M}$ by $\operatorname{diag}\left\{P^{-1}, T^{-1}, I\right\}$ and considering $W=P^{-1}$, $X(\sigma(t))=G(\sigma(t)) W, Y(\sigma(t))=F(\sigma(t)) W, L=T^{-1}$ and $M(\sigma(t))=E_{c}(\sigma(t)) T^{-1}$ results in

$$
\overline{\mathcal{M}}=\left[\begin{array}{ccc}
\tilde{\Gamma}-B_{a} L-V M(\sigma(t))+X(\sigma(t))^{\prime} & P B_{q} \\
* & -2 L & 0 \\
* & * & -\lambda R
\end{array}\right]
$$

with $\bar{\Gamma}=H e\left\{A_{a}(\sigma(t)) W+B_{a} Y(\sigma(t))\right\}+\bar{\lambda} W$, such that $\overline{\mathcal{M}}<0$ also implies (21). Through convexity arguments, if (17) is verified for all matrices $A_{a_{g}}, Y_{g}, M_{g}$ and $X_{g}, g=$ $1 \cdots, 2^{N}$, then $\overline{\mathcal{M}}<0 \forall \sigma(t) \in \mathcal{B}$.

Note also that (17) implies $\dot{\mathbf{V}}(z(t)) \leq-\bar{\lambda} \mathbf{V}(z(t))<$ $0, \forall z(t) \in \Omega(P, 1) \subset S\left(F(\sigma(t)), G(\sigma(t)), u_{0}\right), \forall z(t) \notin$ $\Omega(\eta P, 1)$ and $\forall q(t) \in \Omega(R, 1)$. As a consequence, it follows that $\mathbf{V}(z(t)) \leq e^{-\bar{\lambda} t / 2} \mathbf{V}(z(0)), \forall z(0) \in \Omega(P, 1) \backslash \Omega(\eta P, 1)$. Since the terminal set $\Omega(\eta P, 1)$ is a contracted version of $\Omega(P, 1)$, there exists a $t=t_{1}$ such that $\mathbf{V}\left(z\left(t_{1}\right)\right)=1 / \eta$, i.e. $z\left(t_{1}\right)^{\prime} \eta P z\left(t_{1}\right)=1$. Besides, since $\dot{\mathbf{V}}(z(t))<0$ holds at the boundary of $\Omega(\eta P, 1)$, it follows that all closedloop trajectories enter $\Omega(\eta P, 1)$ and remains confined in it $\forall t \geq t_{1}$.

Constraint (18) ensure that $\Omega(P, 1) \subset \bigcap_{g=1}^{2^{N}} S\left(F_{g}-G_{g}, u_{0}\right)$ and consequently that (16) is verified for all $z(t) \in \Omega(P, 1)$ (Tarbouriech et al., 2011). In the same way, (19) ensure that $\Omega(\eta P, 1) \subset \bigcap_{g=1}^{2^{N}} S\left(F_{g}, u_{0}\right)$, i.e., inside this set no saturation occurs and the closed-loop system is linear.

Based on Theorem 3, an optimization problem is proposed to obtain the gains $F_{g}, E_{c_{g}}$ and to maximize the sets $\Omega(P, 1)$ and/or $\Omega(R, 1)$ (Flores et al., 2009):

$$
\begin{gathered}
\text { OP: } \min \{(1-\kappa) \gamma+\kappa \nu\} \\
\text { subject to } \begin{cases}{\left[\begin{array}{ll}
\gamma & \mathrm{x}_{o}^{\prime} \\
\mathrm{x}_{o} & W
\end{array}\right] \geq 0, o=1, \cdots, f,} \\
\mathrm{~d}_{e}^{\prime} R \mathrm{~d}_{e} \leq \nu, e=1, \cdots, s, \\
\bar{\lambda} \geq \hat{\lambda} \\
\text { Conditions }(17),(18),(19) .\end{cases}
\end{gathered}
$$

The sizes of $\Omega(P, 1)$ and $\Omega(R, 1)$ can be evaluated with respect to polyhedral shape sets $\mathcal{X}_{R}=\operatorname{Co}\left\{\mathrm{x}_{1}, \mathrm{x}_{2}, \cdots, \mathrm{x}_{f}\right\}$ and $\mathcal{D}_{R}=\operatorname{Co}\left\{\mathrm{d}_{1}, \mathrm{~d}_{2}, \cdots, \mathrm{d}_{s}\right\}$, where the vertices $\mathrm{x}_{o} \in$ $\mathbb{R}^{n+n_{c}}, o=1, \cdots, f$ and $\mathrm{d}_{e} \in \mathbb{R}^{p+l}, e=1, \cdots, s$ correspond to directions in which the sets should be maximized and are known a priori. Considering $\alpha=\frac{1}{\sqrt{\gamma}}$ and $\beta=\frac{1}{\sqrt{\nu}}$, then the minimization of $\gamma$ or $\nu$ implies in the maximization of $\alpha \mathcal{X}_{R} \subset \Omega(P, 1)$ or $\beta \mathcal{D}_{R} \subset \Omega(R, 1)$ (Tarbouriech et al., 2011). Note that all optimization subproblems stated in the end of section 3.1 can be addressed by the solution of OP with an appropriate choice of $\kappa \in$ $[0,1]$.

Due to terms $\lambda(-1+3 \eta) W$ in (17) and $\eta W$ in (19), these conditions are LMIs provided that parameters $\lambda$ and $\eta$ are fixed a priori. In this case, a suboptimal solution of OP can be obtained through a grid search in these variables. Finally, restriction $\bar{\lambda} \geq \hat{\lambda}$ imposes a minimum exponential decay rate for the closed-loop trajectories inside $\Omega(P, 1) \backslash$ $\Omega(\eta P, 1)$, therefore acting as a free parameter directly related to closed-loop transient performance. 


\section{NUMERICAL EXAMPLES}

Consider the following MIMO system, adapted from (Flores et al., 2012):

$$
\begin{aligned}
& A(\sigma(t))=\left[\begin{array}{cccc}
0 & 10 & 0 & 1 \\
-100 & -30-\sigma_{1}(t) & 0 & 0 \\
0 & 0 & -37 & 1 \\
0 & 0 & 0 & -19+\sigma_{2}(t)
\end{array}\right] \\
& B=\left[\begin{array}{cc}
0 & -51 \\
17 & 0 \\
0 & -1 \\
-1 & 1
\end{array}\right], B_{d}=\left[\begin{array}{cc}
-1 & 7 \\
11 & 0 \\
3 & 23 \\
1 & 0
\end{array}\right], u_{0}=\left[\begin{array}{c}
10 \\
5
\end{array}\right] \\
& \left.C(\sigma(t))=\left[\begin{array}{llll}
10 & 20-\frac{\sigma_{1}(t)}{5} & 0 & 0 \\
0 & 10+\frac{\sigma_{2}(t)}{5} & 0 & 0
\end{array}\right], \begin{array}{l}
\sigma_{1}(t) \in[0,25] \\
\sigma_{2}(t) \in[0,17
\end{array}\right]
\end{aligned}
$$

The objective is to track sinusoidal references with nominal frequencies $\omega_{0 r}=[2 \pi \sqrt{2} \pi]^{\prime} \mathrm{rad} / \mathrm{s}$ and reject sinusoidal disturbances with $\omega_{0 d}=[0.4 \sqrt{5} \pi \sqrt{2} \pi]^{\prime} \mathrm{rad} / \mathrm{s}$. Controller matrices $A_{c}$ and $B_{c}$ were constructed as in (10) such that a modified resonant controller is introduced at each frequency, with $\omega_{1}=2 \pi \mathrm{rad} / \mathrm{s}, \omega_{2}=\sqrt{2} \pi \mathrm{rad} / \mathrm{s}, \omega_{3}=$ $0.4 \sqrt{5} \pi \mathrm{rad} / \mathrm{s}, \zeta_{h}=0.01$ and $\zeta_{z}=0.99$.

The optimization problem OP is set to maximize $P(\kappa=0)$ in the direction $\mathrm{x}_{1}=\left[\begin{array}{lllll}1 & 1 & 1 & 1 & 0_{1 \times n_{c}}\end{array}\right]^{\prime}$ assuming $\mathrm{d}_{1} R \mathrm{~d}_{1}^{\prime} \leq$ 1 with $\mathrm{d}_{1}=\left[\begin{array}{llll}1 & 0.5 & 0.5 & 0.25\end{array}\right]^{\prime}$. In addition, a transient performance constraint was imposed such that $\bar{\lambda}=4$.

In order to evaluate the proposed method in terms of $\Omega(P, 1)$ size we considered three scenarios: i) scheduled state-feedback and anti-windup gains; ii) state-feedback and anti-windup gains without scheduling $\left(Y_{g}=Y, M_{g}=\right.$ $M$ and $X_{g}=X$ in conditions (17)-(19)); iii) scheduled state-feedback without anti-windup compensation $\left(M_{g}=\right.$ 0 in conditions (17)-(19)). In case i), the optimal solution of OP is obtained for $\eta=2333.9605$ and $\lambda=5.7135 \times 10^{-4}$, resulting in $\alpha=31.5445$. Solving OP for the second and third cases results in $\alpha=9.5045(\eta=711.7099$ and $\left.\lambda=1.8624 \times 10^{-3}\right)$ and $\alpha=3.2762(\eta=1.0239$ and $\lambda=1.9307)$, respectively. Comparing cases i) and ii), it can be seen that the introduction of scheduled gains reduces the synthesis conditions conservativeness, resulting in an $\alpha$ more than three times greater when compared to the one obtained without scheduling. From cases i) and iii), it is clear that the degrees of freedom derived from the introduction of the anti-windup compensator are of paramount importance, increasing the optimal value of $\alpha$, which is the maximum possible enlargement of $\Omega(P, 1)$ in the directions $\mathcal{X}_{R}$, in almost ten times.

Figure 2 illustrate the output and control signals for case i) with $z(0)=31.5469 \mathrm{x}_{1}\left(\right.$ such that $\left.z(0)^{\prime} P z(0)=1\right)$ and scheduling parameters $\sigma_{1}(t)$ and $\sigma_{2}(t)$ depicted in Fig. 3 . References are sinusoidal waves with amplitude $r_{\max }=$ $\left[\begin{array}{ll}1 & 0.5\end{array}\right]^{\prime}$ and a deviation of $+25 \%$ around $\omega_{0 r}$, while disturbances are also sinusoidal signals with the same frequency deviation around $\omega_{0 d}$, amplitudes of $d_{\max }=\left[\begin{array}{ll}0.5 & 0.25\end{array}\right]^{\prime}$ and acting at $t=3 \mathrm{~s}$. Note that $q_{\max }=\left[r_{\max }^{\prime} d_{\max }^{\prime}\right]^{\prime}=\mathrm{d}_{1}$ such that $q_{\max } R q_{\max }^{\prime} \leq 1$. From these figures it is clear that reference tracking and disturbance rejection is achieved even under the occurrence of saturation in both control inputs.

The ultimate boundedness approach proposed in section 3.1 can be verified by the evolution of $z(t)^{\prime} P z(t)$ presented in Fig. 4. Note that after $t=1.2 \mathrm{~s}$ the product $z(t)^{\prime} P z(t)$ is less than $1 / \eta$, i.e. $z(t) \in \Omega(\eta P, 1) \forall t>1.2 \mathrm{~s}$.

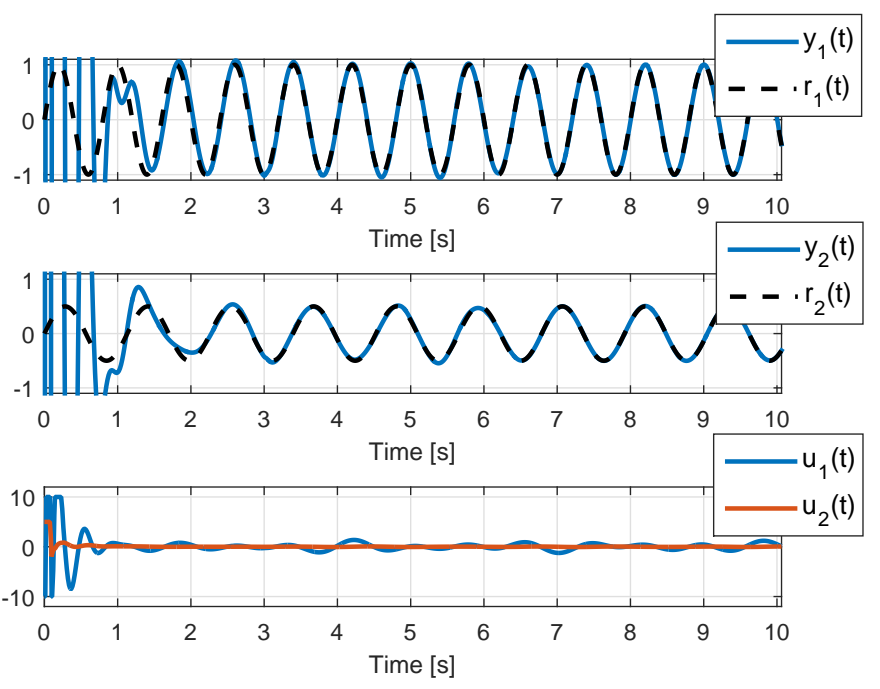

Figure 2. Closed-loop simulation.

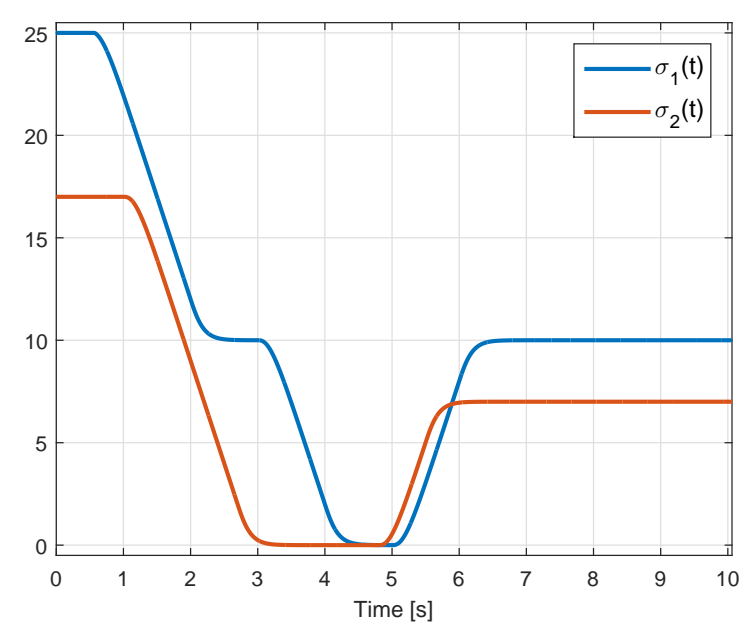

Figure 3. Time-varying parameter $\sigma(t)$.

To illustrate the NRC robustness with respect to frequency variations, the proposed procedure was applied to the standard resonant controller under the same requirements considered in case i). In Fig. 5 is shown the absolute percent tracking error for each reference channel, where one can notice that after $3 \mathrm{~s}$ the RC error is under $30 \%$ and the NRC error is below $12 \%$.

\section{CONCLUSION}

In this paper we addressed the problem of tracking/rejection for LPV MIMO systems subject to input saturation and reference/disturbance frequency variation. Through the state space formulation of the modified resonant controller, the control signal was rewritten in the form of a gain 


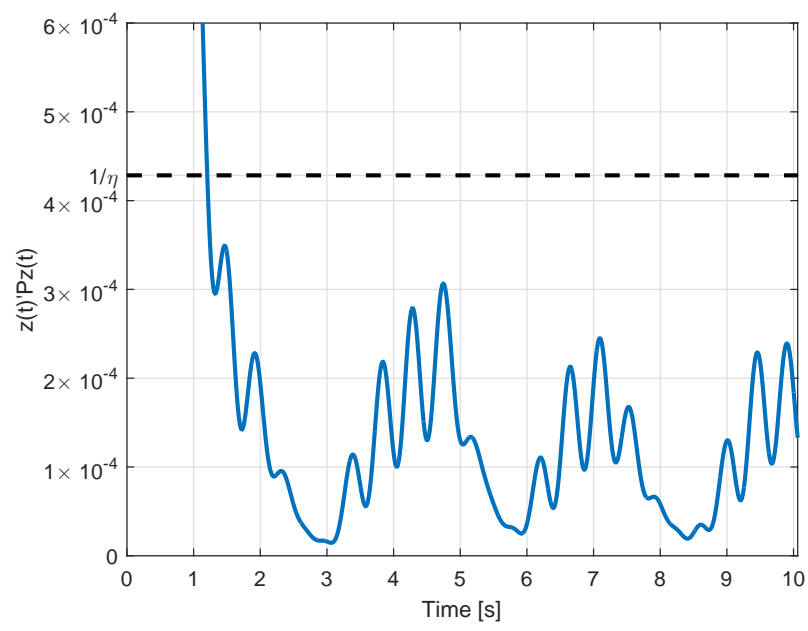

Figure 4. Product $z(t)^{\prime} P z(t)$ over time.

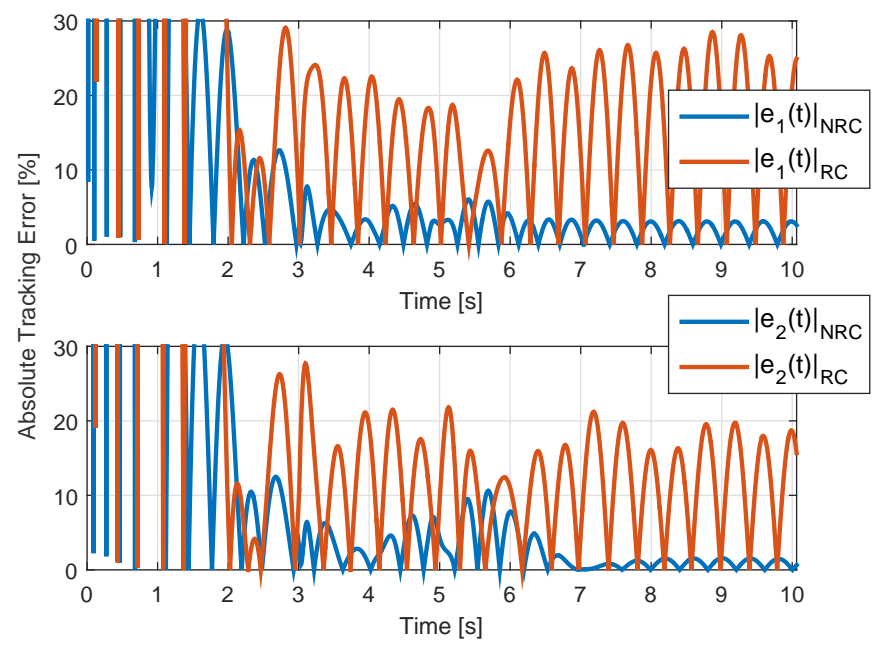

Figure 5. Absolute tracking error.

scheduling augmented state feedback. Also, a scheduled anti-windup compensator was considered to improve the performance under saturation. An ultimate boundedness approach was considered to obtain quasi-LMI conditions to ensure closed-loop stability and convergence of trajectories to an invariant set inside the linearity region. In addition, optimization problems were provided to maximize the set of admissible states and/or exogenous signals. A numerical example illustrated the reduction of conservativeness resulted from gain scheduling and anti-windup compensation, as well as a improved tracking performance of the modified resonant controller when compared to the standard one.

\section{REFERENCES}

Castro, R.S., Salton, A.T., Flores, J.V., Kinnaert, M., and Coutinho, D.F. (2017). Variable frequency resonant controller for load reduction in wind turbines. Control Engineering Practice, 66, 76-88.

Flores, G.P. and Flores, J.V. (2018). Robust design of high order repetitive controllers under control saturation. In 9th IFAC Symposium on Robust Control Design (ROCOND) 2018. IFAC.
Flores, J.V., Eckhard, D., and Salton, A.T. (2016). Modified mimo resonant controller robust to period variation and parametric uncertainty. In Control Applications (CCA), 2016 IEEE Conference on, 1256-1261. IEEE.

Flores, J.V., Gomes da Silva Jr., J.M., Pereira, L.F.A., and Sbarbaro, D.G. (2012). Repetitive control design for mimo systems with saturating actuators. IEEE Transactions on Automatic Control, 57(1), 192-198.

Flores, J.V., Gomes da Silva Jr., J.M., and Sbarbaro, D. (2009). Robust periodic reference tracking for uncertain linear systems subject to control saturations. In Decision and Control, 2009 held jointly with the 2009 28th Chinese Control Conference. CDC/CCC 2009. Proceedings of the 48th IEEE Conference on, 7960-7965. IEEE.

Hara, S., Yamamoto, Y., Omata, T., and Nakano, M. (1988). Repetitive control system: A new type servo system for periodic exogenous signals. IEEE Transactions on automatic control, 33(7), 659-668.

Hoffmann, C. and Werner, H. (2015). A survey of linear parameter-varying control applications validated by experiments or high-fidelity simulations. IEEE Transactions on Control Systems Technology, 23(2), 416-433.

Kang, C.M., Lee, S., and Chung, C.C. (2018). Discretetime lpv $h_{2}$ observer with nonlinear bounded varying parameter and its application to the vehicle state observer. IEEE Transactions on Industrial Electronics, 65(11), 8768-8777.

Li, D. and Ye, Y. (2018). Second-order RC: analysis, augmentation, and anti-frequency-variation for singlephase grid-tied inverter. IET Power Electronics, 11(6), 1128-1134.

Pereira, L.F.A., Flores, J.V., Bonan, G., Coutinho, D.F., and Gomes da Silva Jr., J.M. (2014). Multiple resonant controllers for uninterruptible power supplies - a systematic robust control design approach. IEEE Transactions on Industrial Electronics, 61(3), 1528-1538.

Ramos, G.A. and Costa-Castelló, R. (2013). Optimal antiwindup synthesis for repetitive controllers. Journal of Process Control, 23(8), 1149-1158.

Rugh, W.J. and Shamma, J.S. (2000). Research on gain scheduling. Automatica, 36(10), $1401-1425$.

Steinbuch, M. (2002). Repetitive control for systems with uncertain period-time. Automatica, 38(12), 2103-2109.

Tarbouriech, S., Garcia, G., Gomes da Silva Jr., J.M., and Queinnec, I. (2011). Stability and stabilization of linear systems with saturating actuators. Springer Science \& Business Media.

Tarbouriech, S. and Turner, M. (2009). Anti-windup design: an overview of some recent advances and open problems. IET control theory $\&$ applications, 3(1), 1-19.

Tseng, H.E. and Hrovat, D. (2015). State of the art survey: active and semi-active suspension control. Vehicle system dynamics, 53(7), 1034-1062.

Zhang, B.L., Han, Q.L., and Zhang, X.M. (2017). Recent advances in vibration control of offshore platforms. Nonlinear Dynamics, 89(2), 755-771. 